\title{
Aprendizaje basado en problemas, PBL y aprendizaje basado en equipos, TBL aplicado a las prácticas de laboratorio universitario
}

\section{Josep M. Fernández Novell}

Departamento de Bioquímica y Biomedicina Molecular. Universidad de Barcelona. Barcelona. España.jmfernandeznovell@ub.edu

\begin{abstract}
Resumen
Es sabido que para el desarrollo de una carrera exitosa de un profesional de cualquier Grado en Ciencias es imprescindible poder manejar y resolver circunstancias complejas con los conocimientos, habilidades y competencias adquiridas durante los años de formación universitaria. Si aceptamos que el aprendizaje es el resultado solo de lo que el alumnado hace y piensa llegamos a la conclusión, que sólo se puede lograr a través de muchas horas de prácticas de laboratorio.

De las distintas metodologías activas y diferentes a las clásicas lecciones magistrales, se han utilizado conjuntamente el PBL, aprendizaje basado en problemas, y el TBL, aprendizaje basado en equipos, con éxito en los primeros cursos de los Grados universitarios de Bioquímica y de Bioinformática. Ello ha aportado a nuestros alumnos un mayor nivel de conocimientos que se ha traducido en una mayor capacidad de resolver problemas, analizar datos y, muy importante, pensar de manera crítica.
\end{abstract}

En este trabajo se presentarán y discutirán, con la participación del propio alumnado, la aplicación de dichas metodologías las cuales, están provocando una intensa transformación en la educación universitaria.

Palabras claves: Experimentos, química, primer curso universitario, metodologías activas, PBL y TBL

\section{Introducción}

La falta de interés hacia las carreras científicas, en nuestro país y en Europa, (Fensham, 2004 y Martínez, 1998) acompañada de una cierta actitud negativa hacia las ciencias en nuestros jóvenes estudiantes de secundaria condiciona el momento de elegir una carrera 
universitaria ya que desestiman algunas de ellas por sus contenidos en matemáticas o en física o química.

Actualmente, desde los centros educativos que enseñan las ciencias bajo el modelo STEM, Ciència-Tecnología-Ingeniería-Matemáticas (Sanders, 2009), se intenta revertir esta actitud negativa. Este enfoque requiere el uso de métodos innovadores y alternativos de enseñanza y aprendizaje, tales como proyectos, prácticas de laboratorio y herramientas tecnológicas. La enseñanza se vuelve eminentemente práctica y se aleja del aprender teórico y repetitivo. Se centra en la capacidad de innovar, inventar y resolver problemas de forma creativa.

Un estudio publicado en 2014 (Freeman et al, 2014) realizado en 225 alumnos de Estados Unidos de América, a los cuales se les analizaba bajo la perspectiva del modelo STEM reveló que dicho aprendizaje reduce drásticamente el porcentaje de fracaso universitario y ha llevado a los estudiantes a obtener un mayor nivel de conocimientos y una mayor capacidad para resolver problemas, analizar datos o pensar de manera crítica. Demuestra que los estudiantes no consiguen una positiva comprensión de un terna, de física o de química o de matemáticas o... de ciencias, solo atendiendo a unas explicaciones teóricas de una forma distante.

La educación científica se basa en la indagación (Jiménez Pérez, 2016 y Camacho, 2008). En ella los estudiantes, trabajando en grupos y colaborando entre ellos, participan en la planificación de una investigación para dar respuesta a preguntas y/o problemas de la vida real. Para ello, deben recopilar información y datos de las fuentes a su alcance. Luego, deben proponer explicaciones ajustadas a sus resultados para comunicarlos a los demás grupos de la clase. Deben expresarse usando términos científicos adecuados, tanto por escrito como de forma oral. Y, finalmente, deben participar en discusiones públicas en defensa de su trabajo y dirigir sus explicaciones hacia popularizar la ciencia.

Implementar este tipo de cambios en los primeros cursos universitarios tiene una ventaja, los resultados son a menudo evidentes. Las metodologías activas más utilizadas en el ámbito de las Ciencias son: CBL, de sus siglas en inglés, case based learning o aprendizaje basado en el estudio de casos reales, PBL (Schwartz, 2002), de sus siglas en inglés, problem based learning o aprendizaje basado en problemas, TBL (de Vries, 2018), de sus siglas en inglés, team based learning o aprendizaje basado en equipos y IBL, de sus siglas en inglés inquiry based learning o aprendizaje basado en retos.

De éstas se han utilizado el PBL y el TBL como innovación educativa. Se desarrollan conjuntamente los modelos PBL y TBL en los mismos grupos de estudiantes y al mismo tiempo, para conseguir una mayor motivación y participación por parte del alumnado de primer curso del Grado de Bioquímica de la Universidad de Barcelona (UB) en las asignaturas de Química I y Química II y también en el grado de Bioinformática de la Universitat Pompeu Fabra (UPF) en la asignatura "Aspects of Physical and Organic 
Chemistry", ambas impartidas por el autor desde 2011 en el grado de bioquímica y desde 2016 el de bioinformática, año de su implantación.

Para nuestros universitarios el aprendizaje es el resultado de lo que el estudiante hace y piensa y ello, en los estudios científicos, sólo se puede lograr a través de muchas horas de "prácticas" muy reflexionadas y maduradas. Nuestra aproximación educativa, aprendizaje basado en problemas, no solamente abarca los problemas numéricos en general, sino que la aplicamos a la resolución de problemas y/o casos prácticos en el laboratorio de bioquímica y de bioinformática (PBL) y, siempre, es realizada por un grupo, un equipo, de estudiantes (TBL) rompiendo las fronteras entre los distintos modelos de la metodología activa.

En este artículo se presentan y discuten la puesta en marcha de las metodologías PBL y TBL en ambos grados científicos como apuesta clara para que los estudiantes sean los protagonistas. Finalmente, se presentan las conclusiones/aportaciones del propio alumnado acerca de esta innovación educativa.

\section{Características de los estudiantes del Grado de Bioquímica y del Grado de Bioinformática}

Los diferentes grados científicos dirigen al alumnado a profesiones relacionadas pero distintas y ello queda reflejado en el currículum de cada grado. Así, mientras en los estudios de Bioquímica se potencia la parte experimental con un número importante de créditos de laboratorio, en los estudios de Bioinformática se potencian las prácticas de ordenador.

\subsection{Grado de Bioquímica}

Proporciona una comprensión y uso de las técnicas estándar de la bioquímica y sus aspectos cuantitativos básicos. El contenido del programa de "Química I y II" reúne un conjunto de conceptos básicos y esenciales para desarrollar posteriormente una buena parte de las asignaturas que constituyen el grado de Bioquímica. 1. Bases de las reacciones químicas. 2. Estructura atómica, enlace químico y estructura molecular. 3. Reacciones en disolución acuosa. 4. Equilibrio químico: equilibrio ácido-base, de oxidación-reducción y de precipitación. 5. Biomoléculas (QI)

El alumnado desarrolla la capacidad para comprender y explicar los principios químicos de las reacciones bioquímicas y las técnicas experimentales utilizadas en dicho estudio. 


\subsection{Grado de Bioinformática}

Proporciona una formación intra e interdisciplinaria en temas tanto computacionales como científicos con una sólida formación básica en química. El contenido del programa de "Aspects of Physical and Organic Chemistry (APOC) Aspectos de la química física y orgánica" cubre conceptos fundamentales en física y química orgánica y está distribuido en: 1. Estructura atómica y enlace químico. 2. Termodinámica química. 3. Reacciones de química orgánica. 4. El equilibrio químico: equilibrio ácido-base, de oxidación-reducción y de precipitación (APOC)

El alumnado integra, procesa, gestiona e interpreta datos químicos básicos para una mayor comprensión de los fenómenos biológicos.

\subsection{Competencias semejantes en ambos Grados}

Solo se mencionarán algunas para dar una idea al lector:

- Elaboración y defensa de argumentos y la resolución de problemas dentro de su campo de estudio.

- Transmisión de información, ideas, problemas y soluciones a audiencias especializadas y no especializadas.

- Adquisición de conocimientos químicos a nivel micro y macro, con un especial énfasis en aplicaciones de bioquímica y química orgánica.

- Preparación en tecnologías de frontera y en la utilización de herramientas y recursos de investigación.

Si nos centramos en la adquisición de competencias en el marco de un grado científico, estas solo se pueden lograr experimentando, reconstruyendo soluciones anteriores y preparándose para afrontar nuevos retos en contextos reales.

\section{Metodología}

Con todos estos datos y dada la importancia que el trabajo de laboratorio, de química en este caso, tendrá en el futuro de estos profesionales, se decidió:

Escoger una serie de problemas y/o preguntas relevantes, con un significativo interés por parte de los estudiantes. En la tabla siguiente hay una pequeña lista de dichos problemas que el profesorado discute con los estudiantes para que escojan o presenten una alternativa. 
Estas preguntas deben estar relacionadas, en general, con la bioquímica y la bioinformática y, además, han de tener una posible solución dentro del marco de la investigación "bio" y del trabajo en el laboratorio de química. A veces es difícil que surjan ideas de experimentos que ayuden a solucionarlos.

Las distintas aproximaciones experimentales se llevarán a cabo por grupos de entre 3 y 5 alumnos de la clase (Dennick, 1998). Estos grupos se deberían formar espontáneamente entre el alumnado sin necesidad de participación alguna del profesorado.

\begin{tabular}{|c|c|}
\hline \multicolumn{2}{|c|}{ Trabajos realizados los 2 últimos años con las preguntas que intentan responder } \\
\hline $\begin{array}{l}\text { Hierro en los cereales del desayuno } \\
\text { ¿Cómo medir el hierro de los cereales y } \\
\text { cómo se puede aprovechar? }\end{array}$ & $\begin{array}{l}\text { Ósmosis, difusión y diálisis. } \\
\text { ¿Se puede hacer y explicar la ósmosis } \\
\text { inversa en el laboratorio? }\end{array}$ \\
\hline Sustancias carcinogénicas & Síntesis del ácido acetil salicílico \\
\hline $\begin{array}{l}\text { Seguridad química de dichas sustancias y } \\
\text { su representación tridimensional. }\end{array}$ & $\begin{array}{l}\text { Estudio de la reacción entre el ácido } \\
\text { salicílico y el anhídrido acético. }\end{array}$ \\
\hline Alcalosis respiratoria & Regulación del pH sanguíneo \\
\hline $\begin{array}{l}\text { ¿Cómo medir la capacidad pulmonar? } \\
\text { Diferencias entre fumador/no fumador. }\end{array}$ & $\begin{array}{l}\text { Regulación del equilibrio ácido-base. } \\
\text { Preparación de soluciones tampón. }\end{array}$ \\
\hline $\begin{array}{l}\text { Alimentos } \\
\text { autocalentables/autoenfriables }\end{array}$ & Isótopos radioactivos en medicina \\
\hline Elaboración de dispositivos & órgano en diferentes condiciones? \\
\hline Antioxidantes alimentarios & Leche con y sin lactosa \\
\hline $\begin{array}{l}\text { ¿Cuál es la concentración de polifenoles } \\
\text { en distintas bebidas de té? }\end{array}$ & $\begin{array}{l}\text { ¿Tiene lactasa la leche sin lactosa y cómo } \\
\text { se mide su actividad? }\end{array}$ \\
\hline
\end{tabular}

Los grupos deciden que pregunta o problema resolver y para ello dedican unas 8-10 h de trabajo. Diseñan unos experimentos con todo el material del laboratorio a su disposición y bajo la supervisión del profesor.

Los resultados obtenidos, junto con el guión experimental, forman parte de una memoria escrita final de, un máximo de 10 páginas siguiendo las directrices de un artículo científico: Resumen, Introducción, Metodología, Resultados, Discusión, Conclusiones y Referencias.

El profesor hace una revisión profunda de los resultados obtenidos junto a los componentes de cada grupo. Después de su aprobación, cada grupo puede realizar su presentación oral y pública, frente a toda la clase, con soporte informático, donde se discuten y valoran los resultados obtenidos. En estas presentaciones, cada grupo debe contestar las preguntas y sugerencias formuladas por sus compañer@s de clase. 
Con todo esto presente, el profesor realiza la evaluación de dicha actividad, todas las presentaciones son valoradass por el profesor como un ejercicio más del curso. Se puntuarán, desde la presentación personal, el material de soporte, la memoria final y la respuesta a las preguntas de sus compañeros de clase. Es importante que el alumnado entienda y comparta los resultados de dicha evaluación para valorar su propio trabajo.

La nota final de dicha actividad, que puede ser distinta para cada miembro del grupo, tiene un valor del 10-20\% de la evaluación final de la asignatura correspondiente, "Química" en el Grado de Bioquímica y "APOC" en el Grado de Bioinformática.

Para valorar si el aprendizaje basado en problemas es una actividad que, el alumnado contestó un cuestionario donde, además de preguntas personales y del grado en cuestión, cabe destacar sobre esta actividad:

- ¿Qué dificultades has encontrado durante la realización de tu trabajo?

- ¿Cómo evalúas tu participación en el trabajo experimental, en la preparación de la presentación en ppt y en la preparación del resumen escrito final?

- ¿Cómo evalúas a tus compañer@s de grupo y tu relación con el grupo?

- ¿Cómo se puede mejorar la relación dentro del grupo?

- ¿Qué puntos positivos y negativos de tu trabajo en equipo destacarías?

- ¿Qué cambiarías de tu intervención en un futuro trabajo en equipo?

- ¿Cómo valoras esta actividad?

\section{Resultados}

El resultado de utilizar en la enseñanza universitaria los modelos PBL y TBL al mismo tiempo, convierte el aprendizaje de la química en un proceso más participativo, donde los estudiantes pueden explorar juntos nuevas aproximaciones experimentales. En la Figura 1 i 2 se muestrn distintos momentos de esta actividad educativa. 

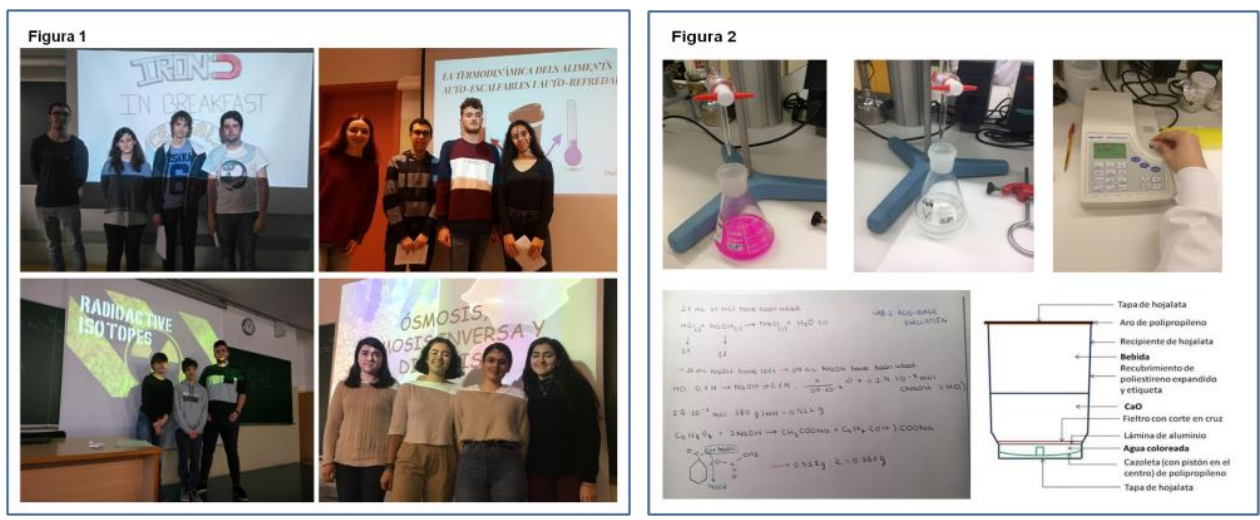

Fig. 1. Se muestran 4 presentaciones: hierro en los cereales, la termodinámica de los alimentos autocalentables y autoenfriables, los isótopos radioactivos y la ósmosis inversa.

Fig. 2. Se muestran distintos momentos en el laboratorio: valoraciones de solución tampón, medida de polifenoles por espectro-fotometría, notas sobre la síntesis de la aspirina de un grupo de alumnods y un dispositivo autocalentable de otro grupo de alumnos.

El lector entenderá que no es necesario, en este punto, comparar los resultados académicos de dicho alumnado con otros que no han realizado dicha actividad porqué para ello debería dividirse, al empezar el curso, la clase en dos grupos, uno con las clases formales y el otro con la actividad propuesta. El autor cree que si se remite a los cursos sobre química dados por él, al mismo tipo de alumnado, en cursos anteriores al 2011-2012, la diferencia de actitud y de aprovechamiento es notorio.

Al terminar el curso, los estudiantes fueron requeridos a responder unas preguntas dirigidas a evaluar esta iniciativa educativa. Entre sus respuestas cabe destacar:

Algunos grupos han tenido difilcultad para encontrar la información necesaria para ampliar el conocimiento sobre el tema a desarrollar. Otros han tenido problemas para distribuirse el trabajo y, algún miembro ha trabajado bastante menos que el resto. También, el hecho de hacer algunas presentaciones en inglés representa cierta dificultad.

Sobre la evaluación personal y del grupo respecto a la preparación y presentación, son más exigentes a la hora de evaluarse a sí mismos y la participación de cada miembro del grupo. pero, en ningún caso la variación ha superado el $20 \%$ de la puntuación del profesor.

Para mejorar el trabajo es necesario analizar los puntos negativos, básicamente, poca comunicación entre los miembros del grupo que repercute en la no correcta distribución del trabajo. También han indicado que, para futuros trabajos, tratarían de organizarse mejor y, sobretot, dedicarían más tiempo a preparar y realizar la presentación. 
Finalmente, 245 participantes del grado de Bioquímica y 62 del grado de Bioinformática, han puntuado esta innovación educativa entre 1-5, siendo 1 (valoración negativa) y 5 (valoración muy positiva). Los resultados recogidos del grado de bioquímica han sido de

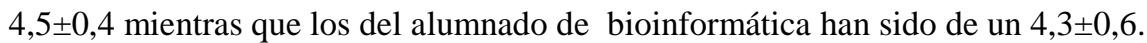

\section{Conclusiones}

- El alumnado del grado de bioinformática presentó, inicialmente, cierta reticencia al trabajo de laboratorio que, posteriormente, pasó a entusiasmo. Mientras que el alumnado del grado de bioquímica siempre ha estado predispuesto al trabajo de laboratorio.

- Esta actividad educativa da la posibilidad de aprender a través de unos proyectos prácticos ideados para responder a las inquietudes científicas del alumnado dentro de un escenario real. Con ello aumenta la motivación e interés por la ciencia en los estudiantes.

- La gran aceptación de dicha metodología entre el alumnado estimula al profesorado a continuar en dicha dirección. La aplicación de dichas metodologías (Deslauriers, 2011) está provocando una intensa transformación en la educación universitaria.

\section{Referencias}

APOC https://www.esci.upf.edu/uploads/imgen/3329-1.pdf (visited 7/03/2019)

Camacho, Hermelinda, Casilla, Darcy, Finol de Franco, Mineira, (2008) La indagación: una estrategia innovadora para el aprendizaje de procesos de investigación. Laurus, vol. 14, nº 26, 284-306.

Dennick, R. G., and Exley, K. (1998). Teaching and learning in groups and teams. Biochem, Education. 26, 111-115.

Deslauriers, L., Schelew, E., and Wieman, C. (2014). Improved learning in a large-enrollment physics class. Science, 332 (6031), 862-864.

De Vries, J., Tweddell, S., and McCarter, R. (2018). Team-Based Learning: Engaging learners and creating team accountability [Electronic version]. Journal of Finnish Iniversities of Applied Sciences. https://uasjournal.fi/in-english/team-based-learning-engaging-learners/

Fensham, P. J. (2004). Beyond Knowledge: Other Scientific Qualities as Outcomes forSchool Science Education. En R.M. Janiuk y E. Samonek-Miciuk (Ed.), Scienceand Technology Education for a Diverse World - dilemmas, needs andpartnerships. International Organization for Science and Technology Education (IOSTE) XIth Symposium Proceedings (pp. 23-25). Lublin, Polland: MariaCurie-SklodowskaUniversityPress. 
Freeman, S., Eddy, S. L., McDonough, M., Smith, M. K., Okoroafor, N., Jordt, H., and Wenderoth, M. P. (2014). Active learning increases student performance in science, engineering, and mathematics. PNAS, 111 (23), 8410-8415.

Jiménez Pérez, G. E., and Tuesta, N. D. (2016). Manual de sesiones de aprendizaje con el enfoque de indagación y alfabetización científica.(VIA EIRL imprenta) Chiclayo. Perú.

Martínez, M., Gros, B., and Romaña, T. (1998). The problem of training in Higher Education. Higher Education in Europe, vol XXIII, n. 4, 483-495.

Química I http://www.ub.edu/grad/plae/AccesInformePD?curs=2018\&codiGiga=361551\& $\underline{\text { idioma }=C A T \& \text { recurs }=\text { publicacio }}($ visited 14/05/2019)

Sanders, M. (2009). "STEM, STEM Education, STEMmania". The Technology Teacher. International Technology Education Association. December, 20-26.

Schwartz, P. (2002). Problem-Based Learning: Case Studies, Experience and Practice. (Kogan Page Editorial) London. United Kingdom.

UB, https://www.ub.edu/web/ub/ca/ (visited 26/06/2019)

UPF, https://www.upf.edu/ (visited 26/06/2019) 